\title{
Les activités ludiques au service de I'enseignement/apprentissage du français ${ }^{(*)}$
}

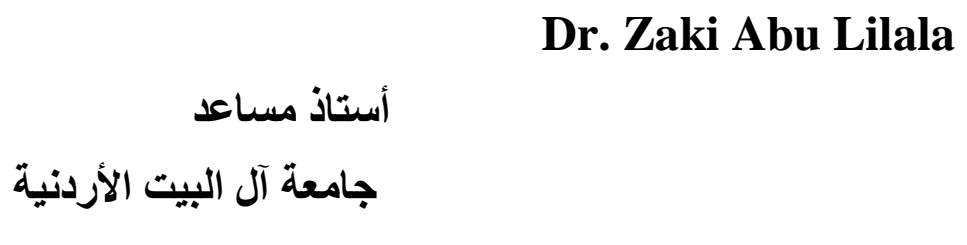

Resume

Les activités ludiques sont considérées comme un moyen d'apprentissage très efficace. Elles représentent un paquet d'avantages pour le processus d'apprentissage d'une langue étrangère. Dans cette recherche, nous allons essayer de voir l'effet de l'utilisation des activités ludiques sur l'enseignement/apprentissage du français dans une classe de FLE en Jordanie. Nous allons voir si ces activités permettent de développer certaines compétences chez l'apprenant comme la lecture (la prononciation) et l'acquisition du lexique. Pour répondre à ces questions et pour mesurer l'impact de l'utilisation de ces activités sur les notes des étudiants, nous avons effectué une étude comparative, longitudinale, sur deux groupes d'apprenants. Un questionnaire a été également réalisé afin de collecter les avis de nos étudiants concernant l'utilisation de ces activités en classe.

Mots-clés:activités ludiques, jeu, acquisition, enseignement/ apprentissage, le français, lecture, lexique, recommandation.

\section{Abstract}

Recreational activities are considered a very effective means of learning. It represents many advantages for the process of learning a foreign language. In this research, we will assess the effect of recreational activities on the teaching/learning of French as a Foreign

${ }^{(*)}$ Bulletin of the Faculty of Arts Volume 79 Issue 6 July 2019 
Language in Jordan. We will assess if these activities help developing certain skills among the learners such as reading (pronunciation) and the acquisition of the lexicon. To answer these questions and to measure the impact of using these activities on students' grades, we conducted a comparative longitudinal study of two groups of students. A questionnaire was also carried out to collect student's opinions concerning the use of these activities in the classroom.

Keywords: recreational activities, game, acquisition, teaching/learning, French language, reading, lexicon, recommendation.

\section{ملغص}

تعتبر الأنشطة الترفيهية وسيلة فعالة جدا للتعلم. وهي تمثل مجموعة من المزايا لعملية تعلم اللغة الأجنبية. في هذا البحث، سنحاول أن نقيّ مدى تأثنير استخدام الأنشطة الترفيهية على تعليم/تعلم اللغة الفرنسية كلغة أجنبية في الأردن. سنكتشف إذا كانت هذه الأنشطة تسمح بنطوير مهارات معينة لدى الطالب مثل القراءة (اللفظ) واكتساب المفردات. للإجابة عن هذه الأسئلة ولقياس مدى تأثير استخدام هذه الأنشطة على تحصيل الطلاب العلمي أجرينا دراسة مقارنة لمجموعتين من الطلاب. كما أنجزنا استنياناً لمعرفة آراء الطلاب حول استخدام هذه الأنشطة في القاعة الدراسية.

الكلمات الدالة: أنشطة ترفيهية، لعبة، اكتساب، تعليم/تعُّم، اللغة الفزنسية، القراعة، معجم (المفردات)، توصية.

\section{Introduction :}

Les didacticiens font leur possible pour trouver de nouveaux moyens qui peuvent participer à augmenter la motivation chez les apprenants, surtout en classe de langue. Ils s'efforcent également pour les pousser à participer activement en classe afin de renforcer l'interaction. Dans un article intitulé Le jeu en classe de FLE : intérêts et pratiques, les auteurs confirment que : «Or, dans l'optique d'une 
approche actionnelle de l'enseignement-apprentissage en classe de FLE, les praticiens sont toujours à la recherche de nouvelles méthodes susceptibles de rendre la classe plus vivante, d'impliquer les apprenants dans leur apprentissage et d'amener ces derniers à utiliser la langue de manière aussi naturelle et authentique que possible. » $(2014: 64)^{1}$.

Les activités ludiques présentent des avantages très variés pour l'acquisition des langues étrangères, mais l'emploi de ces activités en classe, par les enseignants, reste très faible : "Le jeu reste trop peu utilisé à des fins pédagogiques, bien que son utilité soit reconnue par une très large proportion des chercheurs, et ce dans de nombreux domaines, de la pédagogie aux sciences cognitives.» (2014 $: 64)^{2}$.

Dans cette recherche, nous allons essayer de répondre à certain nombre de questions comme : Quels sont les avantages de l'utilisation des activités ludiques dans une classe de FLE en Jordanie ? Comment le ludique peut améliorer le niveau des étudiants ? Le ludique participe-t-il à développer la lecture (la prononciation) et l'acquisition du lexique chez les apprenants ? Et enfin, les activités ludiques facilitent-elles l'apprentissage de la langue étrangère ?

Pour répondre à ces questions, nous avons effectué une étude comparative (longitudinale), faite par nous même, sur deux groupes d'apprenants. Un questionnaire a été également réalisé afin de collecter les avis de nos apprenants concernant l'utilisation des activités ludiques en classe.

Cette recherche vise, entre autres, à affirmer l'importance et l'utilité du ludique pour l'enseignement/apprentissage du français.

${ }^{1 / 2}$ HELME, L., JOURDAN, R., TORTISSIER, K, 2014, « Le jeu en classe de FLE : intérêts et pratiques », Rencontres Pédagogiques du Kansaï. 5 pages, 64-68, on line : http://www.institutfrancais.jp/kansai/files/2014/09/RPK-2014-Article.pdf 
Nous allons aussi voir les avantages des activités ludiques sur tout le processus d'apprentissage et sur les compétences visées durant l'enseignement/apprentissage d'une nouvelle langue étrangère.

Mais avant de parler de notre étude comparative et de notre questionnaire, nous allons essayer de donner un aperçu de ces activités pour éclairer quelques éléments liés à notre sujet de recherche.

\section{Les activités ludiques :}

Dans le petit Larousse illustré 2012, le mot ludique est mentionné comme un adjectif qui signifie : "(du lat. ludus, jeu). Relatif au jeu : Activité ludique. » (2012 : 638). Selon cette définition, très brève, on peut constater que le ludique est lié au jeu. Quant à l'activité ludique, Ameur reprend la définition qui se trouve dans le dictionnaire du français langue étrangère et seconde (Cuq, 2003, p.160) : "Une activité d'apprentissage dite ludique est guidée par des règles de jeu et pratiquée pour le plaisir qu'elle procure ». (2015 : 17).

Selon Harkou, le dictionnaire Le nouveau Petit Robert, (1993, p.1374) définit le jeu comme étant : «Activité physique ou mentale purement gratuite, qui n'a dans la conscience de la personne qui s'y livre d'autre but que le plaisir qu'elle procure. » (2015 : 60).

Boughalem donne la définition suivante au mot jeu : «On peut appeler « jeu » toute activité dont le seul objectif est le plaisir. Le jeu va aider le tout-petit à accéder au «je ». L'enfant va utiliser le jeu pour faire comme s'il était indépendant et il va devenir ainsi peu à peu indépendant. Le jeu va lui permettre de s'affirmer en tant qu'individu, il va jouer à son Autonomie et il va apprendre ainsi à devenir autonome.»(2015 : 12). Douangdara définit le jeu comme toute : "activité divertissante, soumise ou non à des règles, pratiquée par les enfants de manière désintéressée et par les adultes à des fins parfois lucratives ». (2015 : 38). Douangdara distingue trois catégories de jeu : le jeu ludique, le jeu éducatif et le jeu pédagogique. 
(2015 : 38). Selon Douangdara, Silva (2008b) donne au jeu en classe de langue cinq fonctions : la socialisation, l'interaction authentique, la mise en œuvre de stratégies, le développement langagier et cognitif et la motivation. (2015: 39).

En ce qui concerne le jeu ludique, Harkou cite la définition de Denis Girard qui le définit comme étant : "l'activité libre par excellence (n'a ni règles, ni obligatoire, ni esthétisme, il est l'œuvre du plaisir instinctif du joueur). » (2015: 62).

Pour Harkou, le jeu éducatif est un terme ambigu : " car on pourrait se demander s'il est avant tout ludique ou serait-ce purement un exercice à finalité éducative? D'une part, G. Brougère (1995 : 159) rappelle, en effet, que "l'ambiguïté du terme jeu éducatif lui serait constitutive ». D'après lui, dès son apparition et son application à l'école maternelle, le terme a rencontré des contradictions. Il s'agissait pour les institutrices de proposer à l'élève des exercices qui, tout en ayant l'allure $d u$ jeu, leur permettraient d'atteindre l'objectif éducatif qu'elles ont fixé. D'autre part, N. de Grandmont (1997, p.67) contribuait également à clarifier la situation lorsqu'elle met des limites au jeu éducatif. Selon elle, afin de ne pas tomber dans des " jeux d'exercice " qui seraient synonymes de lassitude ou de répétition, il serait souhaitable " d'avoir des objets multivocationnels» c'est-à-dire multiusagers, et de changer de jeu éducatif dès que son fonctionnement aura été saisi par l'apprenant. „ (2015 : 63).

Ameur (2015 : 15) précise que le jeu éducatif n'est pas intrinsèquement différent du jeu ludique. Selon l'auteure, le jeu éducatif représente le premier pas vers la structure. C'est-à-dire, vers l'apprentissage de la règle. (2015: 15). Ameur cite N. de Grandmont qui dit que le jeu éducatif permet de contrôler les acquis, d'évaluer les appris, d'observer le comportement des apprenants, et de favoriser l'acquisition de nouvelles connaissances. Ameur précise que Grandmont pense que le jeu éducatif doit être distrayant et sans 
contraintes perceptibles par le joueur, car l'objectif principal de ce type de jeu est de créer un climat de plaisir. (2015:15).

Enfin, le jeu pédagogique selon Harkou est défini par N. de Grandmont comme tout support qui permet de tester les apprentissages. (2015 : 63). Harkou mentionne que N. de Grandmont indique que dans la plupart des écrits sur le sujet, le jeu pédagogique est confondu très souvent avec le jeu éducatif. (2015 : 64).

Pour Ameur, le jeu pédagogique a pour rôle de faire appel à des connaissances pour en apprendre d'autres ou pour les vérifier et à tester des apprentissages (2015 : 15). Ameur ajoute que le jeu est axé sur le devoir d'apprendre, pour cela, il s'inscrit dans une pédagogie qui est qualifié de «pédagogie de jeu ». (2015: 15).

Pour Renard, il y a des traits qui caractérisent le jeu. Ces traits sont le plaisir, la spontanéité, la gratuité et la créativité. Le jeu, selon Renard, répond à des besoins différents et touche toutes les tranches d'âges. (p.1).

Avant de décider d'employer des activités ludiques en classe, l'enseignant doit prendre en compte certaines exigences comme :

- L'utilisation des activités ludiques en classe ne signifie pas seulement jouer. Boudjemaa pense que ces activités doivent présenter un aspect ludique tout en engageant l'apprenant sur le plan cognitif, car il ne s'agit pas seulement de jouer. (2016:49).

- Pour Boudjemaa, les activités ludiques doivent présenter un défi à relever par les apprenants. (2016:49).

- Pour Helme, Jourdan et Tortissier, avant de choisir des jeux il y a plusieurs questions à se poser. (2014 : 66). Tout d'abord : Quel est l'objectif du jeu ? Selon les auteurs, l'emploi des jeux en classe exige de définir des objectifs d'apprentissage clairs. Pour les auteurs, c'est la question primordiale à se poser. (2014: 66). Puis : Combien ai-je d'apprenants dans ce groupe ? Cela permet à l'enseignant de décider de jouer en équipe ou 
individuellement. (2014 : 66). Ensuite : Quel est leur niveau et leur personnalité ? D'après les auteurs, il ne faut pas confondre les deux. Car, un apprenant qui a un niveau modeste mais ouvert et dynamique peut aider l'enseignant à faire accepter le jeu au groupe, et inversement, certains apprenants de haut niveau n'ont peut-être pas l'esprit réceptif au jeu :

"Il faudra dans tous les cas adapter une règle ou se servir d'un jeu comme prétexte, en accord avec ce que vous connaissez de votre groupe.»(2014: 66).

La question suivante : De combien de temps je dispose ? Les auteurs confirment que certains jeux ne nécessitent que très peu d'explications, mais ce n'est pas toujours le cas. Le niveau du groupe peut aussi influer sur le temps que nous devons consacrer à l'introduction d'un jeu. De plus, les auteurs conseillent de faire attention au comptage des points et des corrections qui sont souvent plus longues qu'on ne l'imagine. (2014: 66).

La cinquième question : De quel espace je dispose ? : "Petite classe? Tables fixes ou mobiles, intérieur, extérieur, utilisation de l'espace de l'école/universitélinstitut complet possible etc... La question de l'espace est liée à la question du mouvement de l'apprenant. Travailler sur la gestuelle, faire lever, dégager la cage thoracique... » (2014: 66).

Enfin : Est-ce que je joue ? Les auteurs disent que participer au jeu peut être très utile pour guider le jeu, corser le challenge, ou le simplifier : " C'est une question délicate qu'il faut adapter en fonction des réponses précédentes... besoin d'un nombre pair ? Pensez aussi que les apprenants aiment souvent jouer avec le professeur, c'est l'occasion de voir d'autres réactions, et de faire tomber un peu plus les barrières, mêmes minimes, de la structure " classe » tableau/public etc... » (2014 : 67).

- Helme, Jourdan et Tortissier conseillent les enseignants de tester les 
jeux avant de les employer dans la classe. Selon eux, l'enseignant peut jouer avec les collègues pour mettre en relief les éventuels problèmes auxquels il sera confronté en classe.

- Boughalem mentionne que l'application de cette méthode (le jeu) exige une bonne organisation de la part de l'enseignant qui doit passer par : bien calculer et délimiter le temps de jeu, préparer et prévoir le matériel nécessaire au jeu, fixer les règles de bonne conduite dès le début et définir clairement les limites et les objectifs du jeu. (2015: 17).

- Selon Boughalem, il faut prendre en considération que le jeu intervienne à un moment précis de l'enseignement/apprentissage de la langue et ne représente pas une leçon à part entière. (2015: 17).

\section{Les contraintes des activités ludiques :}

Selon les experts, quelques contraintes sont présentes pendant l'utilisation du jeu en classe. Ces contraintes touchent plus particulièrement l'enseignant. Elles varient entre le temps consacré au jeu, le déroulement du jeu, le matériel, etc. En ce qui concerne le temps, l'enseignant doit, au préalable, être au courant de la durée de l'activité pour éviter tout problème lié au temps.

L'emploi des activités ludiques en classe risque de rendre les apprenants incontrôlables à cause de la nature du jeu qui motive le public et de son côté compétitif. Pour Boughalem, le jeu risque de provoquer une certaine effervescence à cause de sa nature qui comporte une compétition et un challenge. Les apprenants s'impliquent sans réserve dans le jeu. Pour cela, Boughalem conseille les enseignants de fixer des règles de conduites dès le début. (2015 : 18).

Boughalem fait allusion au matériel en disant que prévoir et préparer le matériel nécessaire au jeu peut être très simple ou demander un réel investissement en temps et en imagination. (2015 : 18). 
Les enseignants hésitent, quelquefois, d'employer les activités ludiques en classe. Ils ont peur de ne pas terminer le programme fixé par l'établissement ou de perdre leur temps ou bien encore parce qu'ils ne maitrisent pas les outils nécessaires à l'emploi du jeu. Boughalem confirme cette idée en indiquant que ces inquiétudes poussent les enseignants à jouer moins ou jamais, car la pression entre le jeu et l'apprentissage est très forte. Boughalem ajoute que la maîtrise des outils numériques pose des difficultés aux enseignants (2015:18).

Bour et Hoyet ajoutent que le jeu peut être parfois un outil contraignant pour l'enseignant. Car, ce dernier a un rôle très chargé. Pour les auteures, l'enseignant a beaucoup de tâches. Il doit cadrer les interactions entre les apprenants pour garantir un véritable apprentissage. De plus, sa place dans le jeu est primordiale, car il est considéré comme un médium d'apprentissage déterminant. L'enseignant doit aussi bien évaluer le temps de déroulement d'un jeu pour ne pas empiéter sur la séance suivante (2012: 28).

Le jeu risque de ne pas plaire à tous les apprenants et certains refusent de jouer. Pour Douangdara, dans ce cas là, l'enseignant doit essayer de varier les jeux et les modalités de travail et les objectifs pédagogiques, et mettre les apprenants à l'aise autant que possible. (2015: 46).

\section{Les avantages des activités ludiques :}

L'emploi des activités ludiques a plusieurs avantages pour l'enseignement/apprentissage des langues étrangères. Nous citons ici quelques uns :

- Les activités ludiques attirent l'attention des apprenants. Dans son mémoire de master, Boudjemaa insiste sur ce point en disant que le ludique attire l'attention des apprenants et les aide à aimer ce qu'ils sont en train d'apprendre. (2016:7).

- Les activités ludiques motivent les apprenants, éveillent leur curiosité, suscitent le désir d'apprendre chez eux et augmentent leur 
confiance. Boudjemaa confirme que le ludique représente un moyen très efficace de motivation chez l'apprenant. (2016:8). Helme, Jourdan, et Tortissier précisent que les activités ludiques étaient considérées comme des activités secondaires et seulement récréatives. Ils ajoutent que ces activités sont pourtant une formidable source de motivation et permettent aux apprenants d'utiliser de façon collaborative et créative l'ensemble de leurs ressources communicatives. (2014:64). Ces auteurs ajoutent que l'avantage principal du jeu lorsque l'on cherche à le mettre en relation avec les théories de la cognition n'est autre que la motivation, notion clef s'il en est, dès lors que l'on parle d'activités ludiques. (2014: 65).

Pour Boughalem, le jeu permet à tous les apprenants de participer, surtout ceux qui sont timides ou anxieux. (2015 : 17). Boughalem ajoute que : "La pratique des activités ludiques favorise la motivation dans les apprentissages. Les apprenants ressentent une confiance en eux, ils ont le désir d'accomplir les taches qui leur sont attribués. »(2015:81).

- Le ludique suscite l'imaginaire et la créativité des apprenants. Selon Boudjemaa, le ludique aide l'enseignant à s'investir et à exploiter l'imaginaire et la créativité de l'apprenant. (2016 : 8). Pour Helme, Jourdan, et Tortissier, les jeux encouragent les apprenants à faire semblant, à simuler, à prétendre ou à imaginer qu'ils sont ailleurs ou qu'ils sont quelqu'un d'autre. (2014: 66).

- Les activités ludiques facilitent l'apprentissage de la langue. Pour Boudjemaa, ces activités font partie des moyens pédagogiques qui servent à faciliter l'apprentissage du FLE et à motiver les apprenants pour aimer cette langue. (2016 : 8). Boughalem confirme ce point en s'appuyant sur un questionnaire destiné aux enseignants de français. Selon Boughalem, les résultats obtenus confirment que le ludique renforce et facilite l'apprentissage, surtout chez les jeunes apprenants. (2015: 81). 
- L'enseignant possède de la liberté pour choisir les activités ludiques les plus adaptées aux objectifs fixés, au niveau de ses apprenants, à leurs besoins et à leurs attentes : "En effet, le jeu permet davantage de liberté et en ce sens accorde une place à l'erreur qui doit être dédramatisée et exploitée en vue de favoriser au mieux l'apprentissage et dans une optique positive. »(Bour \& Hoyet, 2012 : 18).

- Les activités ludiques facilitent la mémorisation ce qui participe à enrichir le vocabulaire et à améliorer les autres compétences chez les apprenants. Pour Boughalem : «L'utilisation du ludique par les enseignants, en particulier ceux du cycle primaire dans diverse pratiques (l'oral, l'écrit, en conjugaison et en vocabulaire ...), trouve un puissant stimulus d'apprentissage, car les apprenants développent leur imagination et leur créativité, ce qui les aide à mémoriser rapidement et correctement les nouvelles connaissances et surtout les nouveaux mots. » (2015 : 81).

- L'emploi de ces activités participe à apporter la vie quotidienne en classe de FLE et sort les apprenants du cadre habituel de cours qui engendre, quelquefois, la monotonie chez eux. Selon Boughalem, le jeu permet de modifier le rythme d'un cours et de relancer l'intérêt des apprenants. (2015:17).

- Selon Helme, Jourdan, et Tortissier, l'introduction du jeu en classe de FLE encourage la prise de parole et l'interaction et constitue une continuité des approches communicatives et actionnelle en offrant un cadre plus ludique. (2014: 65).

- Les jeux améliorent l'interaction et l'échange au sein de la classe ce qui participe à avancer dans le processus d'apprentissage. Pour Boughalem, ces activités favorisent la communication et donnent la possibilité aux apprenants de faire des échanges. (2015 : 81).

- Le ludique facilite la tâche de l'enseignant et lui donne plus de liberté pour observer ses apprenants. Ainsi, l'enseignant peut faire 
évoluer le cours en fonction des lacunes et des besoins qu'il a identifiés. (Renard, p.2).

- Le jeu permet de répondre à des besoins réels et authentiques de communication qui permettront à l'apprenant de s'investir dans l'apprentissage de la langue (Hélène Vanthier citée par Bey, 2014 : 16).

- Le jeu, selon Bour et Hoyet, aide l'enseignant à se débarrasser de quelques problèmes chez l'apprenant. Selon elles, le jeu permet un changement de focalisation de l'objet d'apprentissage vers quelque chose de plus ludique et ceci participe à lever certains blocages chez les apprenants (2012:19).

- Le ludique est un auxiliaire très utile pour l'enseignement des langues. Guerni fait allusion à cette idée en disant que le ludique constitue un moyen pédagogique et un auxiliaire efficace dans l'enseignement des langues, en particulier le français langue étrangère. (Guerni, 23 : 2012)

- Selon Guerni, l'utilisation des activités à caractère ludique en classe favorise, d'après les didacticiens Jean Pierre Cuq et Isabelle Gruca les côtés suivants :

$\rightarrow$ Le développement de l'enseignement des lettres, des phonèmes, des mots, des paragraphes, des textes, etc.

$\rightarrow$ L'acquisition de multiples points de langue comme la grammaire, le lexique, etc.

$\rightarrow$ La possession d'une dimension de plaisir et de détente en matière d'apprentissage (2012: 17).

- Pour Bour et Hoyet, le jeu présente des avantages affectifs. Selon elles, l'apprenant est en mesure de dépasser son égocentrisme grâce aux rôles différents au sein de la situation de jeu (2012 : 18). Ainsi, les auteures pensent que le jeu est un outil puissant de socialisation.

- L'emploi des activités ludiques répond aux exigences et aux objectifs 
fixés par le CECR qui visent l'amélioration de l'enseignement de la langue : "Enfin, l'introduction des jeux en classe de français langue étrangère respecte la perspective de l'apprentissage de la langue par l'exécution des tâches comme le préconise le Cadre Européen Commun de Référence (CECR). Celui-ci accorde toute son importance à l'utilisation ludique, esthétique et poétique de la langue (points 4.3.4 et 4.3.5) dans une démarche résolument actionnelle. » (Renard, p.2).

- Bour et Hoyet précisent que le jeu a une portée culturelle. Selon elles, l'utilisation du jeu permet d'intégrer l'aspect culturel dans l'apprentissage d'une langue. Les auteures ajoutent que l'aspect culturel rend l'apprentissage d'une langue concret, car les apprenants prennent conscience que cet apprentissage leur servira plus tard (pendant les voyages par exemple). (2012:26).

\section{Présentation de l'établissement et du public :}

Nous avons mené notre étude à l'Université Al albayt en Jordanie, au département de langues modernes (langue française) où nous travaillons depuis plusieurs années en tant qu'enseignant de français langue étrangère.

Notre public est composé d'apprenants arabophones (jordaniens) qui sont inscrits à l'université dans le département de français afin d'obtenir une licence de français. Dans les départements de français en Jordanie, les cours se limitent à 4 années universitaires équivalentes au niveau de la licence en France. Nos apprenants n'ont aucun contact avec le français avant leur entrée à l'université.

Les universités jordaniennes fonctionnent à peu près comme les universités américaines et anglaises. Elles appliquent le système de "crédits" (unités de valeurs).

Chaque matière possède un certain nombre d'heures. La plupart en possèdent 3. Au sein des départements de français, les 
étudiants ont un nombre fixe d'heures à suivre pour obtenir un diplôme de français. Il s'agit de 132 heures. Les étudiants peuvent choisir les matières et la répartition du nombre d'heures sur le semestre. Ce système est appliqué dans toutes les universités jordaniennes. L'année universitaire en Jordanie est composée de 3 semestres : le premier semestre (4 mois) de mi-septembre à mijanvier, le deuxième semestre (4 mois) de mi-février à mi-juin et enfin le semestre d'été qui est facultatif pour les étudiants, mais intensif, d'une durée de 2 mois : juillet et août.

Parmi les matières que les apprenants doivent suivre, il y en a deux qui travaillent la lecture. Ces deux matières sont obligatoires pour tous les étudiants et l'enseignant peut y employer des activités ludiques. L'objectif de ces deux cours est de faire acquérir aux apprenants de nouveaux mots en français et de maîtriser la lecture des textes. La maîtrise de la lecture et de la prononciation en français est visée au premier lieu par ces deux cours. L'apprenant prend ces deux matières pendant la troisième année. Ces deux cours sont intitulés :

\section{Lecture 1.}

\section{Lecture avancée.}

Le choix des textes pour ces deux cours se fait par le professeur. L'enseignant choisit les textes en faisant attention à qu'ils soient variés. Les textes choisis sont variés : littéraire, descriptif, narratif, historique, explicatif, informatif, etc.

Durant chaque semestre universitaire en Jordanie, il y a trois examens pour chaque matière :

- le premier examen : 25 notes.

- le deuxième examen : 25 notes.

- l'examen final : 50 notes.

Total $100 \%$

\section{Le déroulement de l'étude :}

Pour répondre aux questions posées au début de notre recherche, 
nous avons choisi de façon aléatoire deux classes différentes. Ces deux classes suivent le même cours qui est lecture 1. Chaque classe est composée de 20 apprenants. Pour réaliser notre étude, nous avons décidé d'utiliser des activités ludiques comme : les cartes et les chansons avec une seule classe qu'on va nommer groupe A. Avec la deuxième classe (groupe $B$ ), nous n'avons utilisé aucune activité ludique pendant le cours. Le semestre suivant et après que les deux groupes ont fini le cours Lecture 1, nous avons regroupé les deux groupes d'apprenants dans la même classe pour qu'ils suivent ensemble le cours Lecture avancée qui a un niveau plus avancé que le cours précédent. Nous avons décidé d'employer des activités ludiques avec tous les apprenants. Nous avons suivi cette démarche afin de répondre aux questions posées à l'introduction et aux questions comme : existe-t-il une différence de niveau entre les deux groupes ? (entre ceux qui ont été familiarisés avec les activités ludiques et ceux qui n'ont pas été -de tout- familiarisés avec ces activités), Quel groupe a fait plus de progrès que l'autre concernant le développement de la lecture et l'acquisition de nouveaux mots ? Quel groupe a obtenu les meilleures notes à la fin de notre deuxième cours Lecture avancée ? Le groupe A ou le groupe B ? Quel est l'avis de nos apprenants concernant l'utilisation des activités ludiques et l'impact de ces derniers sur l'apprentissage de la langue française?

Les activités ludiques qu'on a employées durant le cours Lecture 1 et Lecture avancée étaient de deux types : les cartes et les chansons. Pour les cartes, on avait par exemple un texte traitant comme thème la ferme et le lexique qui a lien avec ce thème. On mettait les cartes qui contiennent séparément un mot et un dessin sur la table. Puis, on demandait à chaque étudiant de venir au tableau pour relier le dessin avec le mot qui convient. Ensuite, on montrait à tout notre public le dessin et le mot qui va avec. L'objectif derrière l'utilisation de ces cartes était de renforcer l'acquisition de nouveaux mots par les étudiants. Nous avons utilisé les cartes avec tous les textes de nos 
deux cours (Lecture 1 et Lecture avancée). On a aussi utilisé les cartes pour aider nos apprenants à découvrir le sens de nouveaux verbes. On présentait séparément des cartes qui contiennent les verbes (manuscrits) et d'autres avec l'mage qui représente ces verbes et c'est à l'étudiant de relier le verbe avec l'image qui le représente.

Pour les chansons, nous les avons utilisées avec la plupart de textes. Par exemple, on avait un texte contenant l'hymne national français. On a fait écouter cet hymne aux apprenants pour plusieurs fois afin qu'ils maîtrisent la prononciation des mots de cet hymne. On avait aussi une petite leçon sur l'alphabet français et on a suivi la même démarche pour que les apprenants maîtrisent la prononciation des lettres, surtout les voyelles. Les étudiants nous parlent, toujours, de la difficulté rencontrée pour prononcer les voyelles. Même les étudiants de quatrième année éprouvent de la difficulté en prononçant ces lettres. Les sons de ces voyelles n'ont pas d'équivalent en arabe.

En plus, nous avons distribué un questionnaire destiné aux étudiants pour avoir leur impression concernant les activités ludiques et leur rôle dans l'enseignement/apprentissage du français.

\section{L'analyse de l'étude :}

Avant de commencer l'analyse de notre étude, il est préférable d'afficher les résultats des étudiants dès le début. Donc, ce sont les résultats des étudiants pour la matière Lecture avancée. Le groupe A (qui a été familiarisé avec les activités ludiques durant la matière Lecture 1) va s'afficher en noir. Pour le groupe B (qui n'a pas été familiarisé avec les activités ludiques durant la matière Lecture 1), il va s'afficher en rouge. Le nombre total des étudiants qui sont inscrits dans le cours Lecture avancée s'élève à 40. 
Zaki Abu Lilala: Les activités ludiques au service

Tableau 1 : notes des apprenants.

\begin{tabular}{|c|c|c|c|}
\hline Apprenant & $\begin{array}{l}\text { La note totale } \\
100 \%\end{array}$ & Apprenant & La note totale $100 \%$ \\
\hline 1 & 84 & 21 & 90 \\
\hline 2 & 92 & 22 & 77 \\
\hline 3 & 85 & 23 & 82 \\
\hline 4 & 88 & 24 & 71 \\
\hline 5 & 86 & 25 & 80 \\
\hline 6 & 90 & 26 & 79 \\
\hline 7 & 95 & 27 & 75 \\
\hline 8 & 93 & 28 & 63 \\
\hline 9 & 90 & 29 & 69 \\
\hline 10 & 80 & 30 & 71 \\
\hline 11 & 82 & 31 & 81 \\
\hline 12 & 81 & 32 & 80 \\
\hline 13 & 94 & 33 & 82 \\
\hline 14 & 97 & 34 & 73 \\
\hline 15 & 90 & 35 & 74 \\
\hline 16 & 91 & 36 & 69 \\
\hline 17 & 83 & 37 & 65 \\
\hline 18 & 86 & 38 & 68 \\
\hline 19 & 89 & 39 & 72 \\
\hline 20 & 96 & 40 & 75 \\
\hline
\end{tabular}

Pour analyser les données de notre étude, nous avons utilisé le logiciel SPSS (Statistical Package for the Social Sciences) version 20. Comme nous l'avons déjà mentionné, 40 apprenants ont fait partie de notre étude : Groupe $\mathrm{A}=20$ étudiants (qui ont déjà été familiarisés 
avec les activités ludiques durant le cours de Lecture 1) et groupe B = 20 étudiants (qui n'ont pas été familiarisés avec les activités ludiques durant le cours de Lecture 1). L'âge minimal des apprenants est 20 ans, l'âge maximal est 23 ans (les apprenants ont entre 20 et 23 ans) et l'âge moyen de tous les apprenants est 21,07 ans. L'écart type est de .526. Pour le sexe des participants, $70 \%$ (28 apprenants) sont des filles et $30 \%$ (12 apprenants) sont des garçons. Le tableau suivant présente les données démographiques de nos apprenants.

Tableau 2 : données démographiques de la classe.

Descriptive Statistics

\begin{tabular}{|c|c|c|c|c|c|}
\hline & $\mathrm{N}$ & Minimum & Maximum & Mean & Std. Deviation \\
\hline age & 40 & 20 & 23 & 21.07 & .526 \\
Valid N (listwise) & 40 & & & & \\
\hline
\end{tabular}

$\mathrm{N}=40$

Le tableau et la figure suivante montrent qu'il y a 3 étudiants qui ont 20 ans (7.5\% de la population de la classe), 32 étudiants qui ont 21 ans (80\% de la population de la classe), 4 ont 22 ans (10\% de la population de la classe) et 1 étudiant a 23 ans ( $2.5 \%$ de la population de la classe).

Tableau 3 : Âge des apprenants

Age

\begin{tabular}{|c|c|c|c|c|c|}
\hline & & Frequency & Percent & Valid Percent & $\begin{array}{c}\text { Cumulative } \\
\text { Percent }\end{array}$ \\
\hline \multirow{5}{*}{ Valid } & 20 & 3 & 7.5 & 7.5 & 7.5 \\
\hline & 21 & 32 & 80.0 & 80.0 & 87.5 \\
\hline & 22 & 4 & 10.0 & 10.0 & 97.5 \\
\hline & 23 & 1 & 2.5 & 2.5 & 100.0 \\
\hline & Total & 40 & 100.0 & 100.0 & \\
\hline
\end{tabular}




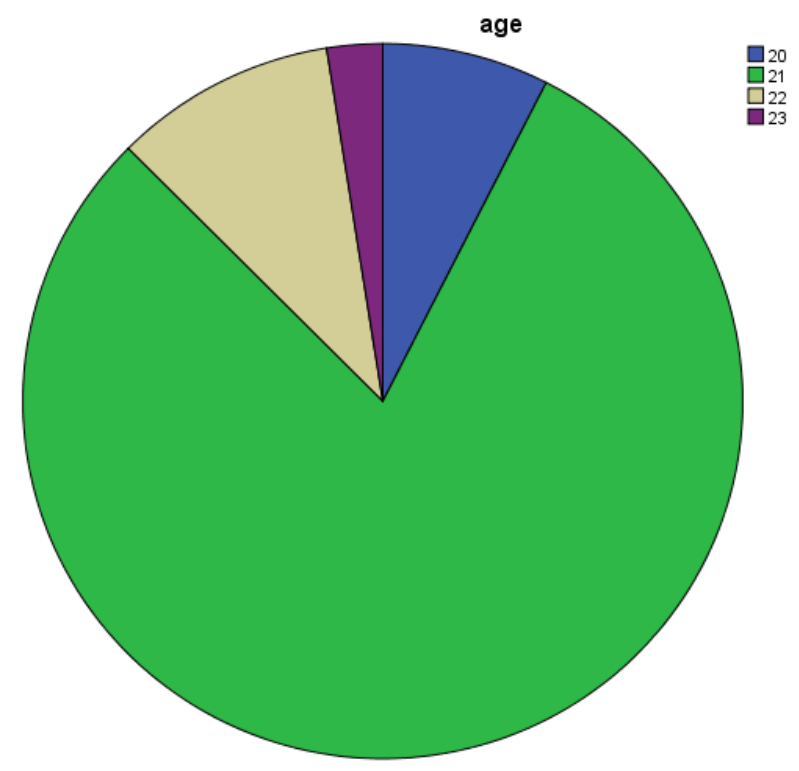

Figure 1 : Âge des apprenants.

La note minimale pour le groupe $\mathrm{A}$ est 80 et la note maximale est 97 , la note moyenne est 88.60, l'écart type est 5.093. Pour le groupe B, la note minimale est 63 et la note maximale est 90 , la note moyenne est 74.80 , l'écart type est 6.646 . Le tableau suivant présente les notes de deux groupes.

Tableau 4 : distribution de notes.

Descriptive Statistics

\begin{tabular}{|c|l|l|l|l|l|}
\hline Game exposure & N & Minimum & Maximum & Mean & $\begin{array}{l}\text { Std. } \\
\text { Deviation }\end{array}$ \\
\hline $\begin{array}{c}\text { yes grade (groupe A) } \\
\text { Valid N (listwise) }\end{array}$ & 20 & 80 & 97 & 88.60 & 5.093 \\
\hline no grade (groupe B) & 20 & 63 & 90 & 74.80 & 6.646 \\
$\quad$ Valid N (listwise) & 20 & & & & \\
\hline
\end{tabular}

$\mathrm{N}=40$ 
Nous voudrions préciser qu'il n'y a pas de relation entre l'âge et les notes obtenues.

En ce qui concerne le genre, il y a une relation très forte entre le genre et l'exposition aux activités ludiques. Les filles ont obtenues les meilleures notes en comparaison avec les garçons. La note moyenne pour les filles est 84.30, leurs notes étaient entre 63 et 97 et l'écart type est 8.56. Pour les garçons, la note moyenne est 73.9, leurs notes étaient entre 65 et 82 et l'écart type est 5.74 .

Tableau 5 : distribution de notes selon le genre.

Descriptive Statistics

\begin{tabular}{|l|l|l|c|}
\hline Gender & Mean & Range & $\begin{array}{c}\text { Std. } \\
\text { Deviation }\end{array}$ \\
\hline Female & 84.30 & $63-97$ & 8.56 \\
\hline Male & 73.9 & $65-82$ & 5.74 \\
\hline
\end{tabular}

$\mathrm{N}=40$

Un t-test (une option dans le logiciel SPSS) a été fait pour mesurer l'effet de l'utilisation des activités ludiques sur les notes des étudiants. Nous avons constaté une différence significative, en ce qui concerne les notes obtenues, entre le groupe A et le groupe B. En fait, le groupe $A$ a obtenu les meilleures notes tandis que le groupe $B$ a obtenu les mauvaises notes. Cela peut être expliqué par le fait que les apprenants qui ont eu l'occasion d'être exposés aux activités ludiques (pendant le cours de Lecture 1) ont obtenu de bonnes notes. L'utilisation de ces activités leur a aidé à améliorer leur niveau, car ils ont été familiarisés avec ces activités et ont réussi à développer les aptitudes nécessaires à la maîtrise de la lecture et à suivre le cours de Lecture avancée sans beaucoup de difficulté et la preuve est les notes qu'ils ont obtenues. Tandis que le groupe B, qui n'a pas d'expérience avec ces activités avant le cours de Lecture avancée, a obtenue les 
mauvaises notes. Le $T$-value est la valeur de T-test. Le $P$ est le niveau significatif du test. Quand le niveau significatif est moins de .05, cela prouve qu'il y a une relation directe entre l'exposition aux activités ludiques et les notes obtenues. Pour notre étude, le niveau significatif est moins de .05 , il est de .000 . Le tableau suivant présente l'effet de l'utilisation des activités ludiques sur les notes obtenues.

Tableau 6 : l'effet de l'emploi des activités ludiques sur les notes.

\begin{tabular}{|l|l|l|l|}
\hline group & Mean & T-value & P \\
\hline $\begin{array}{l}\text { Game exposure } \\
\text { (group A) } \\
\text { (n) 20) }\end{array}$ & 88.60 & 7.371 & .000 \\
\hline $\begin{array}{l}\text { No Game exposure } \\
\text { (group B) } \\
\text { (n= 20) }\end{array}$ & 74.80 & & \\
\hline $\mathbf{N}=\mathbf{4 0 \quad P < . 0 5}$ & & \\
\hline
\end{tabular}

\section{Présentation du questionnaire :}

L'échantillon de notre questionnaire était les 40 apprenants qui ont suivi le cours Lecture avancée. Nous avons distribué le questionnaire après que les étudiants ont passé l'examen final et ont obtenu leurs notes. Notre questionnaire se compose de 5 questions :

1- Pensez-vous que les activités ludiques augmentent la compréhension?

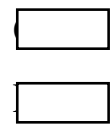

Pourquoi ? 
2- L'utilisation des activités ludiques doit être obligatoire durant les deux matières de lecture?
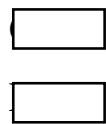

Pourquoi?

3- Pensez-vous que les activités ludiques développent la lecture, la prononciation et l'acquisition de nouveaux mots ?
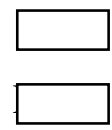

Pourquoi?

- Les activités ludiques facilitent l'apprentissage de la langue française ? 4
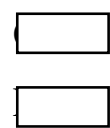

Pourquoi?

- Considérez-vous les activités ludiques comme support motivant ? 5
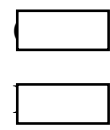

Pourquoi ? Pourquoi ?

L'objectif de notre questionnaire est d'avoir une idée des 
impressions de nos étudiants concernant l'emploi du ludique, son importance, son efficacité et son rôle dans la maîtrise de la lecture (la prononciation) et l'acquisition du lexique.

Pour encourager notre public à participer dans ce questionnaire, nous leur avons expliqué que celui-ci a un objectif scientifique, il vise à améliorer l'enseignement de nos deux cours de lecture et l'anonymat des participants est respecté.

Après avoir distribué le questionnaire, nous avons expliqué la signification de chaque question et les consignes de ce questionnaire. Ensuite, nous avons demandé aux apprenants de prendre leur temps en le remplissant.

\section{Analyse du questionnaire :}

Pour l'analyse de notre questionnaire, nous allons montrer quelques réponses de notre public et n'est pas toutes les réponses, car on a beaucoup d'étudiants et ceci va rendre l'analyse de notre questionnaire très longue.

Pour la première question (Pensez-vous que les activités ludiques augmentent la compréhension ?), tous les étudiants ont répondu par oui. Les réponses étaient de types :

Oui, elles augmentent la compréhension, parce que quand on préparait les textes chez nous à la maison sans consulter un dictionnaire on rencontrait beaucoup de difficultés pour trouver le sens de nouveaux mots, mais en classe en jouant aux cartes, cela nous a facilité la compréhension de nouveaux mots.

Un autre étudiant a précisé :

Les cartes que le professeur nous montre nous aident beaucoup à trouver le sens des mots et ceci facilite la compréhension des textes.

En ce qui concerne la deuxième question (L'utilisation des activités ludiques doit être obligatoire durant les deux matières de lecture?), tous les étudiants, sans exception, étaient avec l'utilisation des activités ludiques dans les deux cours de lecture. Selon eux, ces 
activités présentent beaucoup d'avantages et leur aident à améliorer leur niveau de français. Les étudiants qui n'ont pas eu l'occasion d'avoir des activités ludiques dans le cours de Lecture 1, ont exprimé leur regret, car ils ont l'impression que leurs camarades ont fait plus de progrès qu'eux et qu'ils ont développé plus de compétences en ayant l'occasion d'avoir des activités ludiques dans le cours de Lecture 1. Ils ont constaté une différence de niveau entre les deux groupes (A et B) :

Nos collègues qui ont eu l'occasion d'avoir des jeux pendant le cours de lecture 1 ont beaucoup de chance parce qu'ils ont eu de bonnes notes et ils ont un niveau plus avancé que nous en ce qui concerne la prononciation et l'acquisition de nouveaux mots.

Un autre étudiant a répondu comme le suivant :

Je souhaite qu'on puisse avoir les activités ludiques non seulement pendant ces deux cours, mais aussi dans tous les cours de notre département.

Pour la troisième question (Pensez-vous que les activités ludiques développent la lecture, la prononciation et l'acquisition de nouveaux mots?), tous les apprenants ont répondu par oui. Pour eux, ils essaient d'imiter tout ce qu'ils écoutent dans les chansons et ceci développe la prononciation chez eux. Le jeu des cartes, selon eux, est un outil très efficace pour enrichir le vocabulaire :

Quand on écoute les chansons, on a l'occasion d'écouter des natifs qui chantent et qui prononcent les mots français correctement, on essaie de les imiter et cela nous a beaucoup aidé à améliorer notre prononciation.

Un autre a dit :

Le jeu de cartes est un moyen très amusant et très efficace qui nous aide à acquérir de nouveaux mots. On oublie rarement les mots qu'on a trouvés à l'aide de ces cartes.

Concernant la quatrième question (Les activités ludiques facilitent l'apprentissage de la langue française?), tout notre public a 
répondu par oui. Pour eux, l'emploi de ces activités leur a aidé à comprendre beaucoup de mots et leur emploi dans le contexte. Les apprenants pensent aussi que ces activités leur aident à mémoriser et à imiter la prononciation et tout cela a participé à diminuer le nombre de difficultés qu'ils rencontrent pendant l'apprentissage du français.

Les jeux développent non seulement la prononciation, mais aussi le vocabulaire. On a acquis beaucoup de nouveaux mots.

D'autres étudiants ont répondu par :

Les activités ludiques nous ont permis d'acquérir de nouveaux mots. Ces mots sont très utiles, surtout quand on nous demande d'écrire un texte portant sur n'importe quel thème. On a maintenant une réserve de mots qu'on peut utiliser aux bons moments.

Pour eux, la maîtrise de la prononciation des mots conduit directement vers la maîtrise de la lecture des textes. Un étudiant a précisé :

On a beaucoup souffert pendant la première année avec la prononciation, car c'est une nouvelle langue pour nous. Maintenant, avec les cours de laboratoire de langues pendant la première année et les cours de lecture, notre prononciation des mots s'est améliorée, en conséquence de tout cela, la lecture des textes s'est améliorée aussi.

Pour la dernière question (Considérez-vous les activités ludiques comme support motivant?), tous les étudiants ont répondu par oui. Car, l'emploi de ces activités change le mode de cours qu'ils ont l'habitude d'avoir. Quelquefois, il y a des cours qui engendrent la monotonie pour les apprenants, mais les activités ludiques changent l'atmosphère de la classe et attirent l'attention des apprenants. Selon eux, même les apprenants qui sont modestes et timides essaient de participer, car ces activités suscitent l'enthousiasme. D'après les apprenants, les activités ludiques permettent de créer une atmosphère motivante et appropriée à l'apprentissage :

Je trouve ces activités très motivantes, parce qu'on apprend en s'amusant. 
Pour les étudiants, l'emploi de ces activités les sort du cadre habituel de cours qui provoque souvent l'ennui et la monotonie.

Tout le monde aime le changement. Ces activités ont changé le rythme de nos cours. Ce rythme est devenu très intéressant et plus attirant.

D'après les résultats de ce questionnaire, nous pouvons dire qu'il y a un accord à l'unanimité concernant les bienfaits et les avantages des activités ludiques pour l'apprentissage du français. Tous les apprenants, sans exception, étaient avec l'emploi de ces activités pendant les cours de lecture. Les apprenants pensent aussi que les activités ludiques développent la prononciation, la lecture et enrichissent le vocabulaire. Ils sont d'accord aussi avec l'idée que ces activités facilitent l'apprentissage de la langue française. Pour eux, le ludique constitue un élément motivant pour l'apprentissage.

\section{Conclusion :}

Les activités ludiques ont un rôle non négligeable dans l'enseignement/apprentissage des langues étrangères en général et dans l'enseignement du français en particulier. Les avantages de ces activités sont nombreux que ce soit pour l'apprenant ou pour l'enseignant. L'utilisation du ludique en classe de langue développe plusieurs compétences chez l'apprenant et facilite le travail de l'enseignant : " L'interprétation et l'analyse des résultats de cette enquête et de l'expérimentation nous ont orientées vers des points bien précis qui sont les suivants:

- L'activité ludique est une méthode très utile et importante dans la structure et aussi l'assimilation de nouvelles connaissances bien que l'enseignement de la langue française qui est une langue étrangère peut largement en bénéficier. » (Boughalem, 2015 : 81).

D'après les résultats de notre étude comparative sur deux groupes d'apprenants et les résultats de notre questionnaire, nous pouvons dire que les activités ludiques ont beaucoup d'effets positifs sur le processus d'apprentissage de la langue française. Les experts 
encouragent l'utilisation de ces activités en classe à condition de respecter certaines règles. Des règles comme l'adaptation de ces activités à l'âge des apprenants, à leurs besoins, aux objectifs de l'enseignant et de l'établissement, etc.

Selon les résultats de notre étude comparative et de notre questionnaire, les activités ludiques:

Ont de nombreux avantages dans une classe de FLE, améliorent le niveau des apprenants, participent à développer des aptitudes comme la maîtrise de la lecture, l'acquisition de nouveaux mots, facilitent l'apprentissage de la langue, augmentent la compréhension, sont motivantes et tous les apprenants préfèrent leur utilisation pendant les cours de lecture et les autres cours de notre département. Pour Bour et Hoyet : "Les situations ludiques sont également l'occasion de faire répéter et réutiliser de manière naturelle des structures ou du vocabulaire ainsi que d'améliorer les compétences de prononciation et de compréhension par une mise en situation. » (2012 : 19).

Conformément aux résultats tirés de nos études (notre étude comparative et le questionnaire), nous recommandons l'emploi des activités ludiques pour la majeure partie de cours de notre département pour leurs avantages sur l'ensemble du processus d'apprentissage de la langue.

D'autres recommandations sont également nécessaires comme le besoin de former les enseignants à l'emploi des activités ludiques. Boughalem insiste sur cette idée en disant : «Or, la maîtrise des outils numériques peut poser des difficultés aux enseignants : ils sont généralement moins coutumiers de ce qu'est un jeu que ne le sont leurs élèves. Prensky ${ }^{24}$ indique qu'il y a nécessité d'assurer une formation des enseignants pour la pratique de certains jeux ou univers, pour lesquels les « digital natives » auraient plus de pratique. » 2015 : 19). Bour et Hoyet insistent également sur le besoin d'une formation destinée aux enseignants afin qu'ils puissent tirer les plus 
grands profits des activités ludiques : «Aussi, H. Silva précise qu'il est essentiel de former les enseignants à une utilisation consciente du jeu et de l'intégrer de façon cohérente dans une séquence d'apprentissage. » (2012:18).

Il est recommandé aussi de faire un équilibre, par les enseignants, entre les activités ludiques et les autres moyens qui sont entre les mains. Car, il ne faut pas intensifier l'emploi de ces activités au détriment d'autres moyens disponibles et vice-versa : "Cependant, nous avons pu soulever certaines limites de son utilisation en classe qu'il convient de ne pas négliger et qui nous permettent d'affirmer que le jeu ne saurait être la seule entrée lors de séquences en langues. Nous pensons donc qu'il faudrait faire un compromis et varier les situations d'apprentissage... »(Bour \& Hoyet, 2012 : 35). 


\section{Bibliographie :}

Ameur, M. 2015. Le jeu de l'oie comme activité de motivation à l'expression orale et l'enrichissement du vocabulaire dans une

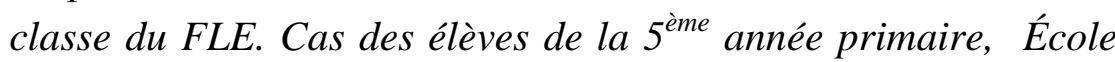
$1^{\text {er }}$ novembre 54, Magra - Msila, mémoire de master, Université de M'Sila (Algérie), 73 pages.

Bey, F E. 2014. Pour une approche ludico-actionnelle pour le développement d'une compétence interculturelle dans la classe de FLE, mémoire de master, Université d'El-Oued -Algérie-, 90 pages.

Boudjemaa, I. 2016. Le rôle des activités ludiques comme facteur de motivation dans l'enseignement du FLE (Cas des apprenants de la $2^{\text {ème }}$ AM École OTHMANE SAAD), mémoire de master, Université de Larbi Tebessi -Tébessa- (Algérie), 60 pages.

Boughalem, S. 2015. La place du ludique dans l'apprentissage du français langue étrangère chez les apprenants de la troisième année primaire, mémoire de master, Université Abou Bekr Belkaïd -Tlemcen-, (Algérie), 102 pages.

Bour, C. and Hoyet, C. 2012. En quoi le jeu facilite-t-il l'apprentissage d'une langue étrangère à l'école primaire?, mémoire de master, Université Montpellier II, France, 61 pages.

Douangdara, L. 2015. Motiver l'apprentissage du FLE par le jeu. Expériences avec des élèves adolescents scolarisés au Laos, mémoire de master, Université Stendhal -Grenoble 3-, France, 101 pages.

Guerni, S. 2012. L'amélioration de la prise de parole à travers les activités ludiques. Cas des apprenants de $5^{\text {ème }}$ année primaire de l'école "les frères Osmani » à Féliache BISKRA, mémoire de master, Université Mohamed Kheider - Biskra (Algérie), 85 pages. 
Harkou, L. 2015. «Pour l'utilisation de l'activité ludique en Algérie dans la classe de FLE », in: Synergies Algérie n ${ }^{\circ} 22$, pp. 59-71.

Helme, L. Jourdan, R. and Tortissier, K. 2014. « Le jeu en classe de FLE : intérêts et pratiques », Rencontres Pédagogiques du Kansaï. 5 pages, 64-68, on line :

http://www.institutfrancais.jp/kansai/files/2014/09/RPK-2014Article.pdf

Le petit Larousse illustré, 2012. Larousse, Paris, 1910 pages.

Renard, C. « Les activités ludiques en classe de français langue étrangère. L'art d'instruire et d'apprendre avec plaisir », on line : https://www.scribd.com/document/142002778/Le-Jeu-en-Classede-FLE 